\title{
Estudio experimental de la estabilidad y propiedades termofísicas de los nanolubricantes PEG 400-CuO, PEG 400-Grafeno y PEG 400-CuO/Grafeno
}

\author{
Experimental study of the stability and thermophysical properties of PEG400 with \\ $\mathrm{CuO}$ and $\mathrm{G}$ as a biodegradable nanolubricant
}

\author{
Jaime Felipe Moreno-Mendoza ${ }^{1}$, Juan Felipe Santa-Marín ${ }^{1}$, Robison Buitrago-Sierra ${ }^{1}$, Karen Cacua ${ }^{1 *}$
}

Forma de citar: Moreno-Mendoza, J. F., Santa-Marín, J. F., Buitrago-Sierra, R., \& Cacua, K. (2021). Estudio experimental de la estabilidad y propiedades termofísicas de los nanolubricantes PEG 400CuO, PEG 400-Grafeno y PEG 400-CuO/Grafeno. Revista Fuentes, el reventón energético, 19(1), 75-84. https://doi.org/10.18273/revfue.v19n1-2021007

\begin{abstract}
Resumen
La adición de nanomateriales a los lubricantes convencionales se ha presentado como una alternativa para modificar sus propiedades termofísicas y tribológicas, buscando incrementar su rendimiento. Este estudio presenta los resultados de la evaluación experimental de estabilidad, conductividad térmica y viscosidad dinámica de los nanolubricantes PEG400-CuO, PEG400-Grafeno y PEG400-CuO/Grafeno. Los nanolubricantes se prepararon por el método de dos pasos y se utilizaron dos concentraciones 0.1 y $0.5 \%$ p/p de cada tipo de nanomaterial. Los resultados experimentales mostraron que las dispersiones con menor aglomeración y sedimentación durante el tiempo de evaluación fueron las preparadas con $0.1 \% \mathrm{p} / \mathrm{p}$ de grafeno. La conductividad térmica de la dispersión PEG400-CuO no presentó diferencias significativas con respecto a la conductividad del PEG400. Sin embargo, la conductividad para las dispersiones PEG400-Grafeno y PEG400-CuO/Grafeno $(0.5 \%$ p/p), incrementó hasta un $13.5 \%$ y $5.2 \%$, respectivamente. La viscosidad dinámica de las dispersiones con una concentración de $0.1 \%$ p/p no presentó cambios significativos con respecto al PEG400, mientras que la viscosidad de la dispersión G-PEG para la concentración de $0.5 \%$ p/p fue superior que la del PEG400 para todas las temperaturas evaluadas.
\end{abstract}

Palabras claves: polietilenglicol, PEG400, nanolubricantes biodegradables, conductividad térmica, viscosidad dinámica, estabilidad, nanofluidos.

\begin{abstract}
The addition of nanomaterials to conventional lubricants has been presented as an alternative to modify their thermophysical and tribological properties, seeking to increase their performance. This study presents the results of the experimental evaluation of stability, thermal conductivity, and dynamic viscosity of the nanolubricants, PEG400-CuO, PEG400-Graphene and PEG400-CuO / Graphene. The nanolubricants were prepared by the twostep method and two concentrations 0.1 and $0.5 \mathrm{wt} . \%$ of each type of nanomaterial were used. The experimental results showed that the dispersions with less agglomeration and sedimentation during the evaluation time were those prepared with $0.1 \mathrm{wt}$ \% of graphene. The thermal conductivity of the PEG400-CuO dispersion did not show significant differences compared to the conductivity of PEG400. However, the conductivity for the PEG400Graphene and PEG400-CuO / Graphene dispersions (0.5 wt./), increased up to $13.5 \%$ and 5.2\%, respectively. The dynamic viscosity of the dispersions with a concentration of $0.1 \mathrm{wt} . \%$ did not show significant changes with respect to PEG400, while the viscosity of the PEG400-Graphene dispersion (0.5 wt.\%) was higher than that of PEG400 for all evaluated temperatures.
\end{abstract}

Keywords: polyethylene glycol, PEG400, biodegradable nanolubricants, thermal conductivity, dynamic viscosity, stability, nanofluids.

\footnotetext{
${ }^{1}$ Grupo de Investigación Materiales Avanzados y Energía- MATyER Instituto Tecnológico Metropolitano (ITM), Facultad de Ingeniería, Medellín, Colombia. *karencacua@itm.edu.co
} 


\section{Introducción}

Los lubricantes son compuestos de aceite y aditivos usados principalmente para lubricar y refrigerar el contacto en sistemas mecánicos. Los lubricantes pueden ser clasificados en función del origen del aceite base como minerales, sintéticos o biológicos. Actualmente, son usados en diferentes sectores de la industria, $\mathrm{y}$ al igual que los combustibles son derivados de petróleo, generando así un crecimiento exponencial en el consumo de combustibles fósiles, debido al crecimiento industrial que se vive actualmente en el mundo (Kotia et al., 2018). Esta situación conlleva a que los investigadores estén buscando alternativas para sustituir estos lubricantes no renovables por aceites de origen vegetal o animal, los cuales son netamente biodegradables y amigables con el medio ambiente (Dhanola \& Garg, 2020b; Osama et al., 2018).

Los aceites sintéticos biodegradables cuentan con propiedades tales como, excelente lubricidad, alto punto de inflamación, alto índice de viscosidad y buena capacidad de cizallamiento (Darminesh et al., 2017; Soni \& Agarwal, 2014). Sin embargo, los lubricantes biodegradables presentan algunos problemas como la sensibilidad a la degradación termo oxidativa (Dhanola \& Garg, 2020b). Adicionalmente, tienen baja viscosidad y propiedades corrosivas que limitan su uso como lubricante industrial (Kim \& Kim, 2015; Wu et al., 2019; Zhang et al., 2014). A pesar de estas propiedades adversas, los lubricantes biodegradables podrían ser una fuente alternativa para la sustitución de los lubricantes derivados del petróleo y constituyen una oportunidad de investigación (Dhanola \& Garg, 2020b).

Entre los lubricantes biodegradables se encuentran por ejemplo el aceite de coco (Koshy et al., 2015), aceite de jojoba (Suthar et al., 2020), aceite de palma (Gulzar et al., 2015), aceite de ricino (Bhaumik et al., 2020), aceite de canola (Dhanola \& Garg, 2020a) y aceite de girasol (Cortes et al., 2020). Adicionalmente encontramos lubricantes a base de fluidos biodegradables como el agua la cual cuenta una excelente capacidad de enfriamiento (Huo et al., 2020) y el polietilenglicol (PEG), el cual cuenta con propiedades físicas y químicas únicas como baja inflamabilidad, miscibilidad en el agua, alta lubricidad, resistencia a la oxidación y a altas temperaturas (Chen et al., 2005; Hu et al., 2018; Marcos et al., 2019; Yapici et al., 2014).

La adición de nanomateriales en los lubricantes biodegradables ha mostrado resultados positivos en su rendimiento y propiedades termofísicas. Cuando se adicionan nanomateriales la conductividad térmica aumenta, se reduce el coeficiente de fricción y el desgaste entre superficies en contacto (Akilu et al., 2017; Darminesh et al., 2017; Mehrali et al., 2014). Además, la adición de los nanomateriales permite incrementar la viscosidad y que esta sea más estable a cambios de temperatura, lo cual proporciona una mayor resistencia de la película lubricante que se forma entre las piezas en movimiento relativo en los equipos (Pownraj \& Valan Arasu, 2020a).

Entre los nanomateriales más usados para la preparación de nanolubricantes biodegradables se encuentran los nanomateriales carbonosos como los nanotubos de carbono $\left(\mathrm{CNT}_{\mathrm{S}}\right)$, grafeno $(\mathrm{G})$, diamante (Azman \& Samion, 2019; Babar \& Ali, 2019) y óxidos metálicos como óxido de cobre $(\mathrm{CuO})$, dióxido de titanio $\left(\mathrm{TiO}_{2}\right)$ y óxido de silicio $\left(\mathrm{Si}_{2}\right)$ (Darminesh et al., 2017). Sin embargo, en los últimos años se ha incrementado el uso de nanomateriales híbridos, los cuales consisten en la combinación de dos o más nanomateriales que modifican sus propiedades a través de su interacción y que al ser dispersados en un mismo fluido base, generan un efecto diferente en sus propiedades comparado con el efecto de los nanomateriales individuales. Este tipo de nanolubricantes ha llamado la atención debido a que los nanomateriales híbridos presentan propiedades fisicoquímicas superiores a las de los componentes individuales. Esta combinación de propiedades físicas y químicas de diferentes materiales permite un aumento en el rendimiento térmico y tribológico de los lubricantes, gracias al efecto sinérgico otorgado la combinación de dos tipos de nanomateriales (Sarkar et al., 2015).

Sin embargo, la estabilidad de los nanolubricantes es uno de los principales desafíos para su uso en diferentes aplicaciones. La aglomeración y posterior sedimentación de los nanomateriales hace que las propiedades de los nanomateriales cambien con el tiempo y pueden producir efectos negativos en el coeficiente de fricción (Azman \& Samion, 2019; Babar \& Ali, 2019; Mello et al., 2020; Yu \& Xie, 2012).

En los últimos años los investigadores han desarrollado técnicas para mejorar la estabilidad en los nanolubricantes tanto biodegradables como minerales. El uso de tensoactivos, control de $\mathrm{pH}$, y vibración ultrasónica son una de las técnicas utilizadas en la preparación de nanolubricantes para aumentar su estabilidad (Dhanola \& Garg, 2020a; Hemmat Esfe et al., 2018; Nabil et al., 2017). 
En este trabajo se evaluó experimentalmente la estabilidad, conductividad térmica y viscosidad dinámica de los nanolubricantes PEG400-CuO, PEG400-Grafeno y PEG400-CuO/Grafeno, con el fin de determinar el efecto de la concentración y el tipo de nanomaterial en estas importantes propiedades.

\section{Metodología}

\section{Materiales y métodos}

Nanoláminas de grafeno (G) y nanopartículas de óxido de cobre $\mathrm{CuO}$ comerciales fueron utilizadas para la preparación de las dispersiones, sus propiedades se muestran en la Tabla 1. Los nanomateriales fueron caracterizados utilizando microscopía electrónica de barrido (SEM, por sus siglas en inglés) y espectroscopía de dispersión de energía de rayos X (EDS, por sus siglas en inglés), utilizando un microscopio electrónico de barrido (JEOL JSM7100F). Para la preparación de las dispersiones se utilizaron los nanomateriales sin ninguna modificación y para el caso del nanomaterial híbrido se realizó una mezcla 50:50 de grafeno y $\mathrm{CuO}$. Como fluido base se utilizó polietilenglicol (PEG400), sus propiedades se muestran también en la Tabla 1. El PEG400 fue caracterizado utilizando espectroscopía de infrarrojo por transformada de Fourier (FTIR, por sus siglas en inglés), utilizando un espectrofotómetro de infrarrojo IRTracer-100 con longitudes de onda entre 800 y $4000 \mathrm{~cm}^{-1}$, con el fin de identificar sus grupos funcionales característicos.

La preparación de las dispersiones se realizó por el método de dos pasos (Pownraj \& Valan Arasu, 2020b; Yu \& Xie, 2012). En este método, las nanopartículas son previamente obtenidas y posteriormente dispersadas en el fluido base. Para este caso se utilizaron dos concentraciones $(0.1$, y $0.5 \%$ p $/ \mathrm{p})$. Para la dispersión se utilizó una sonda de ultrasonido (Qsonica Q500) con una frecuencia de $20 \mathrm{kHz}$, amplitud de $30 \%$ y en modo $2 \mathrm{~s}$ encendido- $2 \mathrm{~s}$ apagado, durante 30 minutos.

Tabla 1. Propiedades de los nanomateriales y el PEG400.

\begin{tabular}{|c|c|c|c|c|}
\hline Material & $\begin{array}{c}\text { Densidad } \\
\left(\mathrm{g} / \mathrm{cm}^{3}\right)\end{array}$ & $\begin{array}{c}\text { Tamaño } \\
\text { promedio } \\
(\mathrm{nm})\end{array}$ & $\begin{array}{c}\text { Viscosidad } \\
\text { (cSt) }\end{array}$ & Fabricante \\
\hline $\begin{array}{l}\text { Óxido de cobre } \\
(\mathrm{CuO})\end{array}$ & 6.31 & $<50$ & NA & $\begin{array}{l}\text { Sigma- } \\
\text { Aldrich }\end{array}$ \\
\hline Grafeno (G) & 2267 & $6 \pm 8$ & NA & $\begin{array}{l}\text { Sigma- } \\
\text { Aldrich }\end{array}$ \\
\hline $\begin{array}{l}\text { Polietilenglicol } \\
\qquad 400\end{array}$ & 1.13 & NA & 84 & $\begin{array}{c}\text { Nicotine } \\
\text { River }\end{array}$ \\
\hline
\end{tabular}

\section{Evaluación de la estabilidad de las dispersiones}

Para evaluar la estabilidad de las dispersiones se utilizó la variación de absorbancia UV-visible en el tiempo, utilizando un espectrofotómetro UV-visible (Agilent 8453 Agilent Technologies 2000; Alemania) e inspección visual. La absorbancia se midió a la máxima longitud de onda de absorción determinada experimentalmente, $292 \mathrm{~nm}$ para el nanolubricante PEG400-CuO y $319 \mathrm{~nm}$ para los nanolubricantes PEG400-Grafeno y PEG400-CuO/Grafeno.

\section{Evaluación de conductividad térmica y viscosidad}

La conductividad térmica y la viscosidad se midieron a $25^{\circ} \mathrm{C}, 35^{\circ} \mathrm{C}$ y $45^{\circ} \mathrm{C}$, esta medición se realizó inmediatamente después de preparar los nanofluidos. Para controlar la temperatura de las dispersiones se utilizó un baño termostático (LAUDA Alpha RA8), recirculando agua en un sistema de intercambio de calor. La conductividad térmica de las dispersiones se midió utilizando un medidor de hilo caliente transitorio (KD2 Pro, Decagon Devices, Inc), usando un beaker de $20 \mathrm{ml}$ y con un tiempo de espera entre cada medida de 15 minutos para que la temperatura se estabilice.

Para medir la viscosidad de las dispersiones se utilizó un viscosímetro programable BROOKFIELD modelo LVDV-II+ PRO. Todas las dispersiones fueron preparadas por duplicado para verificar los resultados. En este caso la viscosidad del fluido base no varió con la modificación de la tasa de corte, dado que el fluido es Newtoniano a pesar de ser modificado con los nanomateriales. Este comportamiento se genera por las bajas concentraciones de nanomateriales usadas en este trabajo.

\section{Resultados}

En las figuras 1, 2 y 3 se muestran las imágenes SEMEDS de los materiales $\mathrm{CuO}$, Grafeno y $\mathrm{CuO} /$ Grafeno. En las imágenes SEM se observa la forma cuasi-esférica del $\mathrm{CuO}$ con un tamaño promedio de $50 \mathrm{~nm}$. En el caso del grafeno se muestra su morfología laminar, con un espesor de lámina aproximado de $6 \pm 8 \mathrm{~nm}$. Los espectros obtenidos por EDS indican los elementos predominantes cobre y oxígeno para el $\mathrm{CuO}$ y carbono y oxígeno para el caso del grafeno. En el caso de la mezcla entre $\mathrm{CuO}$ y $\mathrm{G}$ no se observa cambio de morfología de los nanomateriales originales al estar mezclados. Otros picos no se identifican, debido a que corresponden con el 
oro usado como recubrimiento durante la observación. En este caso no se presentan los resultados cuantitativos, dado que el volumen de interacción en nanomateriales es muy superior al tamaño del objeto de interés y podría generar resultados incoherentes.

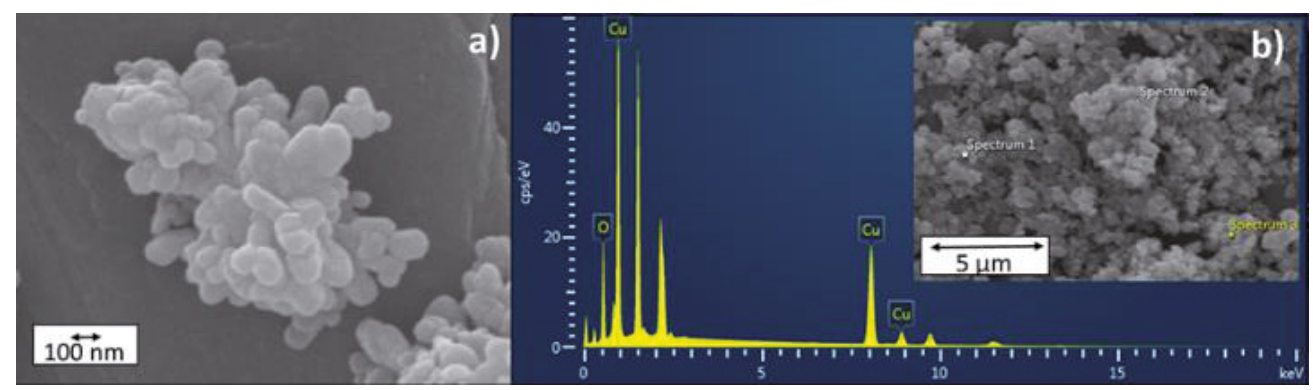

Figura 1. Nanopartículas de cobre a). Microscopía electrónica de barrido SEM para el CuO, b). Espectroscopía de dispersión de energía EDS para el $\mathrm{CuO}$.

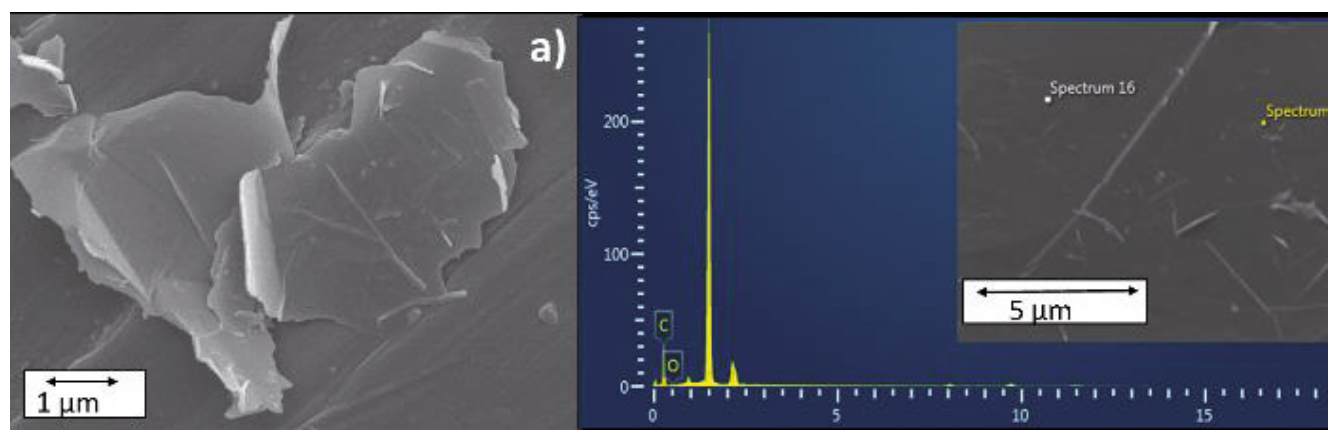

Figura 2. Nanoláminas de grafeno a). Microscopía electrónica de barrido SEM para el Grafeno, b). Espectroscopía de dispersión de energía EDS para el Grafeno.

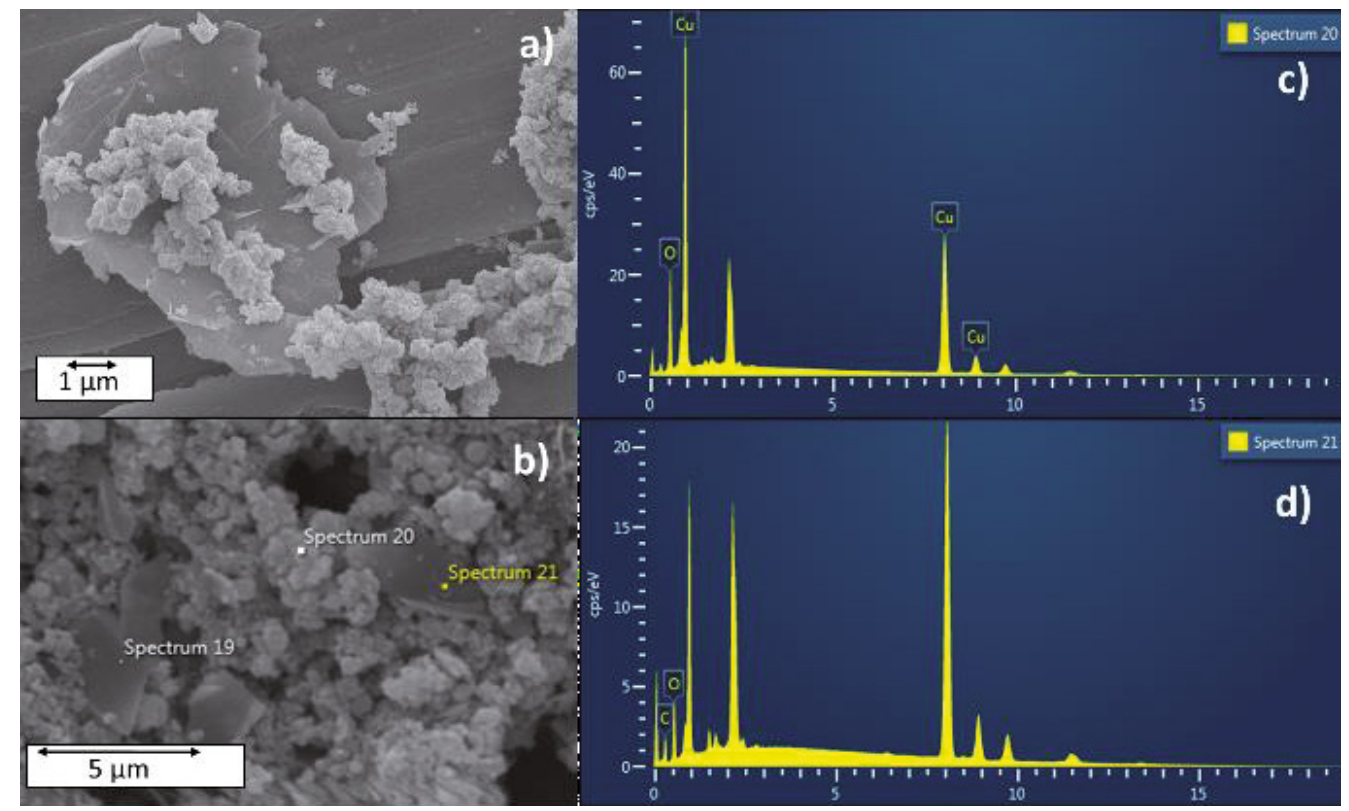

Figura 3. Nanomateriales híbridos a,b). Microscopía electrónica de barrido SEM para el CuO/Grafeno, c,d). Espectroscopía de dispersión de energía EDS para $\mathrm{CuO} /$ Grafeno 
En la Figura 4, se muestra el espectro FTIR del PEG400 en donde se pueden observar varios picos característicos del PEG400, siendo los más representativos los asociados a las vibraciones de estiramiento de alquilo $\mathrm{C}-\mathrm{H}$ presentados alrededor de las longitudes de onda de $2882 \mathrm{~cm}^{-1}$, los estiramientos de C-H están ubicados alrededor de 1457, 1352, 1298 y $1250 \mathrm{~cm}^{-1}$ y a una longitud de onda de $1109 \mathrm{~cm}^{-1}$ pertenece a los estiramientos de C-O-C.

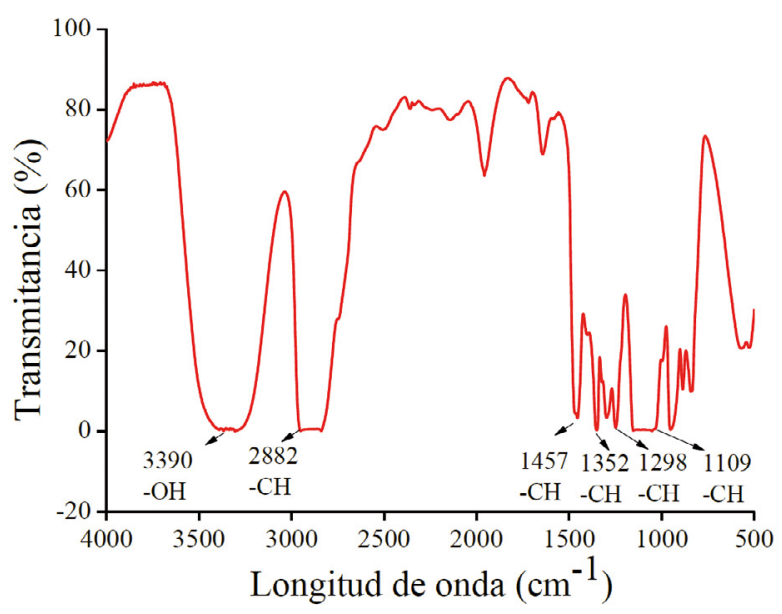

Figura 4. Espectro FTIR del PEG400.

Los resultados obtenidos de los picos característicos para los grupos funcionales del PEG400, son coherentes con los encontrados en la literatura (Alemdar et al., 2005; León et al., 2017).

\section{Estabilidad}

En la Figura 5 y Figura 6 se muestran los resultados de variación de absorbancia UV-vis con el tiempo para las dispersiones preparadas con concentraciones de $0.1 \%$ y $0.5 \%$ en peso, respectivamente. En este caso se observa que, de acuerdo con la ley de Beer-Lambert, al aumentar la concentración de las nanopartículas, la absorbancia aumenta ligeramente. No se observa otro cambio significativo en la estabilidad del nanofluido en el tiempo.

En las Figuras 5 y 6 se observa una tendencia decreciente de la absorbancia con el tiempo para todas las dispersiones preparadas, adicionalmente, este comportamiento fue similar para ambas concentraciones. Sin embargo, se observa que la absorbancia para todas las dispersiones tuvo una disminución mayor para la concentración de $0.5 \%$ $\mathrm{p} / \mathrm{p}$, indicando que el incremento en la concentración afecta la estabilidad. Así mismo, las dispersiones de grafeno presentaron la mayor absorbancia en el tiempo de seguimiento con respecto a las otras dispersiones, mientras que las dispersiones con $\mathrm{CuO}$ presentaron la menor absorbancia, indicando que esta dispersión presenta la menor estabilidad. Este resultado está acorde con lo reportado por Hou et al., (2020).

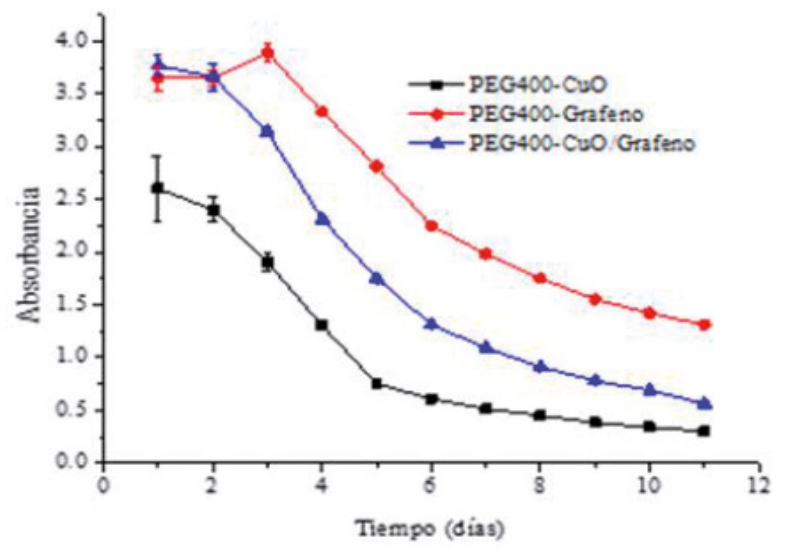

Figura 5. Variación de la absorbancia con el tiempo para las dispersiones de $0.1 \% \mathrm{p} / \mathrm{p} \mathrm{CuO}, \mathrm{G}$ y $\mathrm{CuO}-\mathrm{G}$.

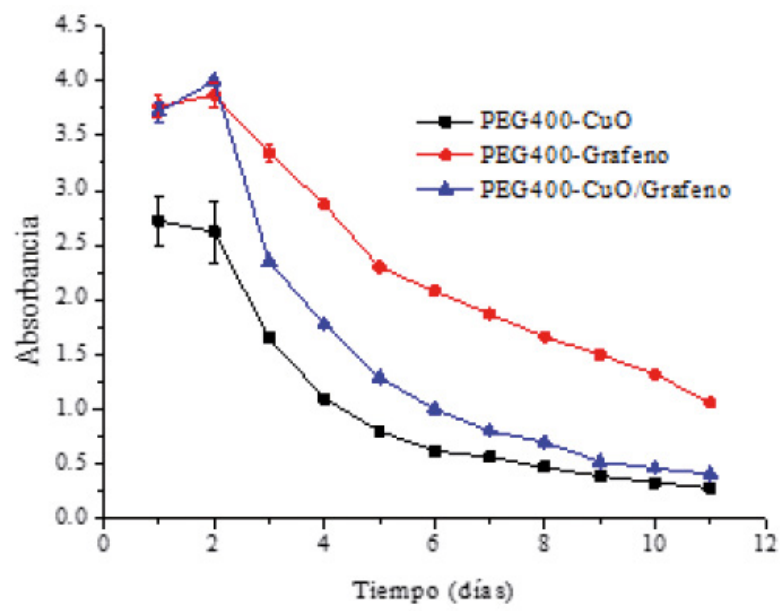

Figura 6. Variación de la absorbancia con el tiempo para las dispersiones de $0.5 \% \mathrm{p} / \mathrm{p} \mathrm{CuO}, \mathrm{G}$ y $\mathrm{CuO}-\mathrm{G}$.

En la Figura 7 se presentan las imágenes de inspección visual para las dispersiones PEG400-CuO, PEG400Grafeno y PEG400-CuO/Grafeno, preparadas con una concentración de $0.1 \%$ p/p y en la Figura 8 , las imágenes de las dispersiones preparadas con una concentración de $0.5 \% \mathrm{p} / \mathrm{p}$. 


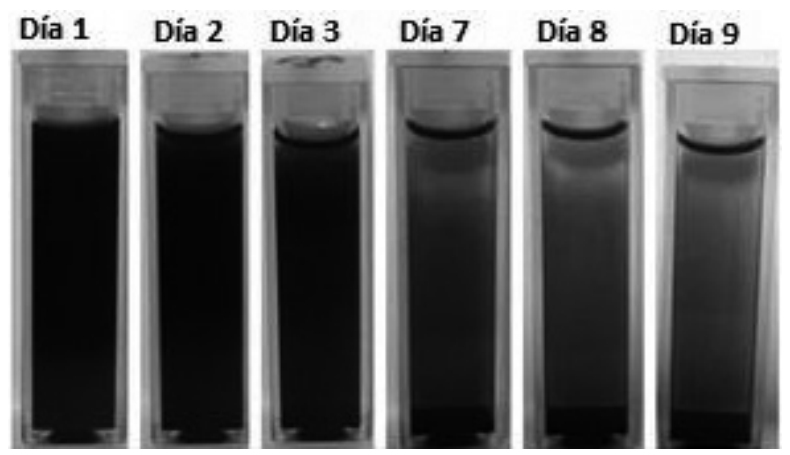

(a)

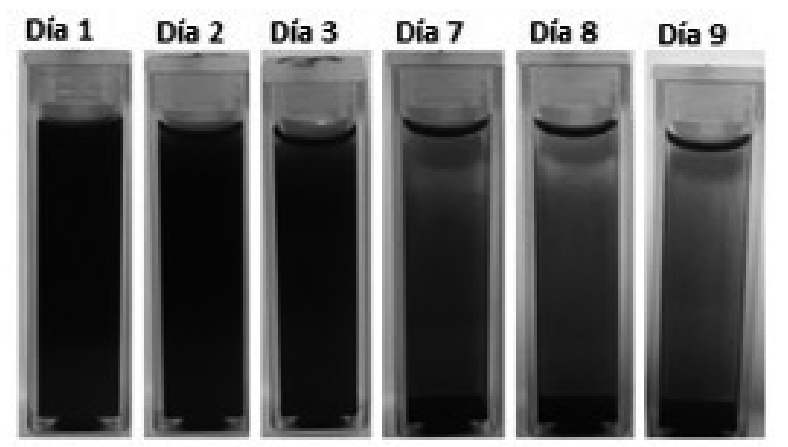

(b)

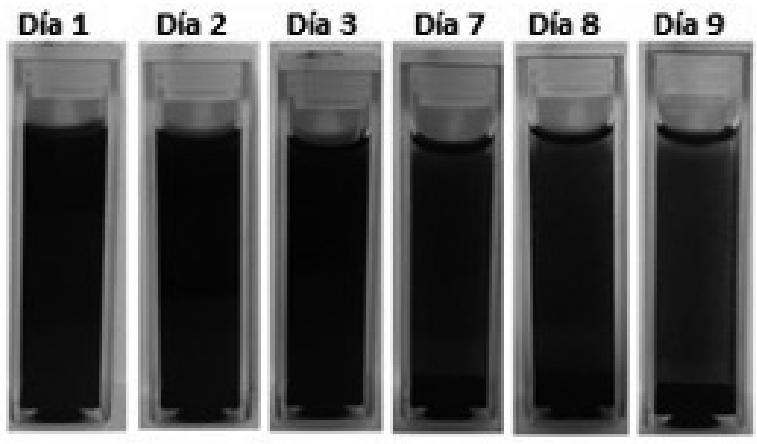

(c)

Figura 7. Inspección visual para la dispersión en PEG400 (a) $\mathrm{CuO}$, (b) Grafeno y (c) CuO-Grafeno (0.1\% en peso ).

Las imágenes de seguimiento visual muestran que los nanomateriales después de la dispersión no presentan sedimentación. Sin embargo, después del día 2 los nanomateriales comienzan a sedimentar y es más evidente para el caso de las dispersiones con $\mathrm{CuO}$. Por su parte, las dispersiones de grafeno para ambas concentraciones (Figuras 8 y 11) presentaron la menor sedimentación en el tiempo de seguimiento, resultados que están de acuerdo con los resultados de seguimiento por absorbancia UV-Vis.

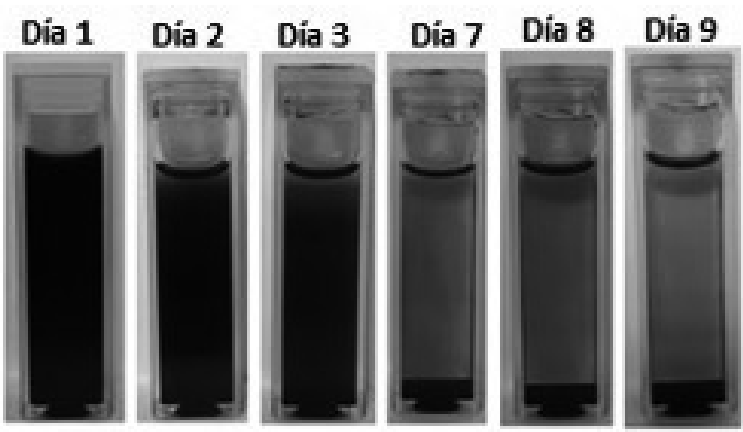

(a)

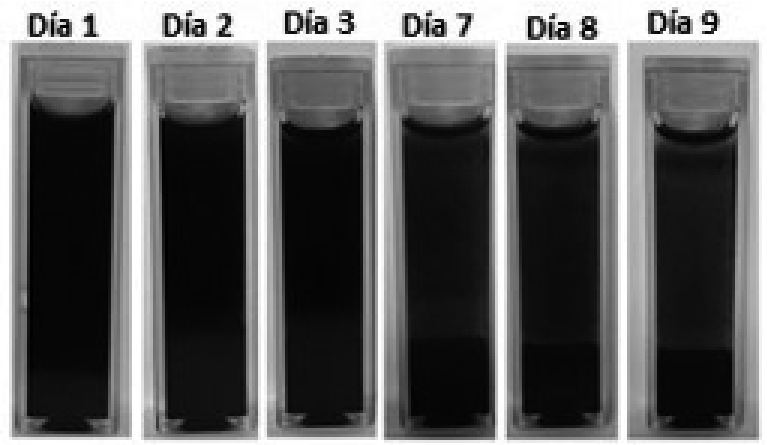

(b)

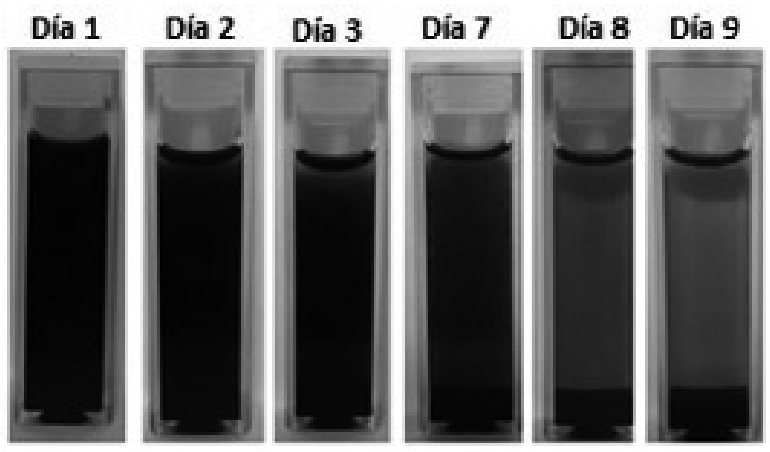

(c)

Figura 8. Inspección visual para la dispersión de grafeno en PEG400 ( $0.5 \%$ en peso $)$.

\section{Conductividad térmica}

Los resultados experimentales de la conductividad térmica obtenidos para el fluido base PEG400 y los nanolubricantes PEG400-CuO, PEG400-Grafeno y PEG400-CuO/Grafeno para las concentraciones de $0.1 \%$ y $0.5 \%$ en peso se muestran en la Figura 9 y Figura 14, respectivamente. 


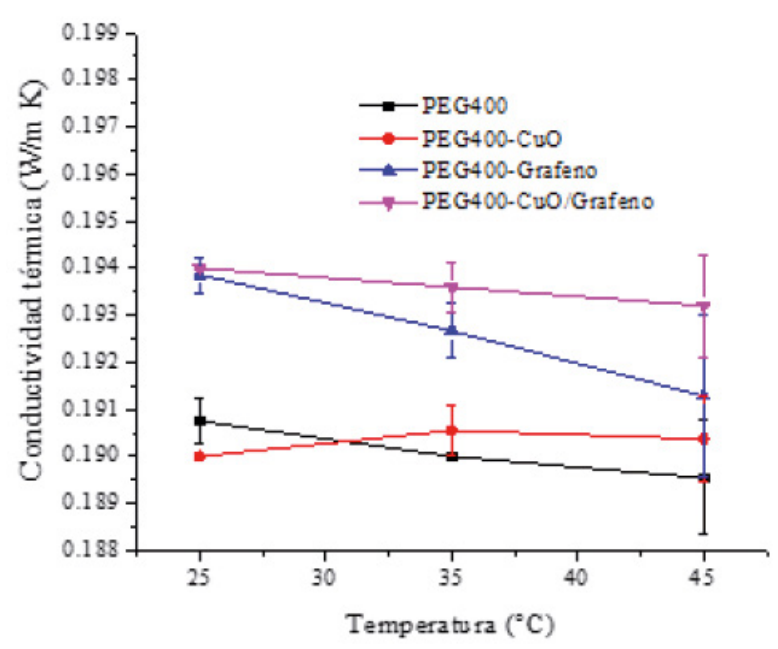

Figura 9. Conductividad térmica en función de la temperatura para las dispersiones con una concentración de $0.1 \% \mathrm{p} / \mathrm{p}$.

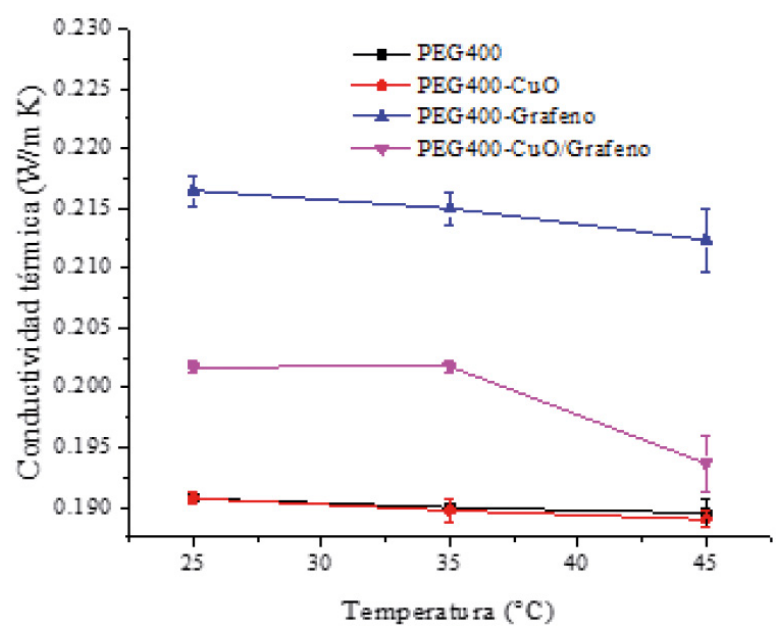

Figura 10. Conductividad térmica en función de la temperatura para las dispersiones con una concentración de $0.5 \% \mathrm{p} / \mathrm{p}$.

En la Figura 9 se observa que la dispersión de $\mathrm{CuO}$ tiene un comportamiento anómalo pues a $25^{\circ} \mathrm{C}$ presentó una conductividad térmica menor que la del PEG400 y en las otras dos temperaturas no presentó diferencias con respecto a la conductividad del PEG400 a pesar de la alta conductividad de este nanomaterial.

Este comportamiento puede deberse a la baja estabilidad de este nanomaterial como se muestra en los resultados de absorbancia e inspección visual. En este caso debido a la alta concentración y a la alta temperatura se favorecen las colisiones y, por lo tanto, existe mayor probabilidad de aglomeración y posterior sedimentación de los nanomateriales, generándose una disminución en la conductividad térmica del nanofluido. Por otro lado, en la literatura (Marcos et al., 2018) han reportado un comportamiento similar a los resultados obtenidos en este trabajo.

En el caso de los nanolubricantes PEG400-Grafeno y PEG400-CuO/Grafeno se muestra un incremento en la conductividad térmica a $25^{\circ} \mathrm{C}$ de $2 \%$ con respecto al PEG400. Así mismo, se identificó que al incrementar la temperatura hasta $45^{\circ} \mathrm{C}$ se muestran desviaciones estándar más altas, no permitiendo identificar cambios significativos en la conductividad térmica con respecto al fluido base.

$\mathrm{Al}$ aumentar la concentración de los nanomateriales hasta $0.5 \% \mathrm{p} / \mathrm{p}$ (Figura 14) no se encontraron diferencias entre la conductividad térmica de PEG400 y la dispersión de $\mathrm{CuO}$. Sin embargo, para los nanolubricantes PEG400Grafeno se encontró un incremento máximo de $13.5 \%$ a $25^{\circ} \mathrm{C}$ con respecto al PEG00 puro y para el nanolubricante $\mathrm{PEG} 400-\mathrm{CuO} / \mathrm{Grafeno}$ se obtuvo un incremento máximo de $5.8 \%$ a $35^{\circ} \mathrm{C}$.

Los resultados de conductividad térmica muestran que al incrementar la concentración de nanomateriales se presenta un mayor incremento en esta propiedad (Geng et al., 2019).

\section{Viscosidad dinámica}

Los resultados experimentales de la viscosidad en función de la temperatura para el fluido base PEG400 y los nanolubricantes PEG400-CuO, PEG400-Grafeno y PEG400-CuO/Grafeno, se muestran en las Figura 11 y 12.

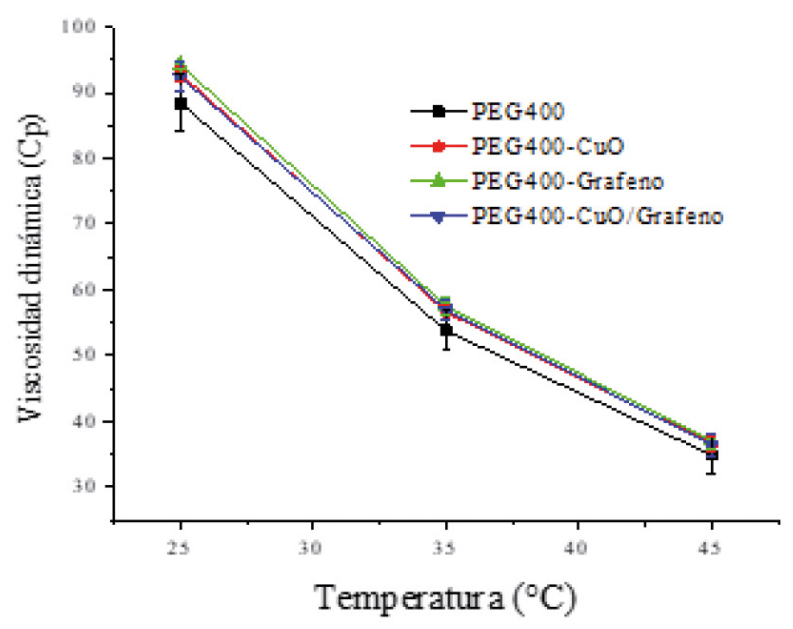

Figura 11. Viscosidad en función de la temperatura para las concentraciones de $0.1 \% \mathrm{p} / \mathrm{p}$. 


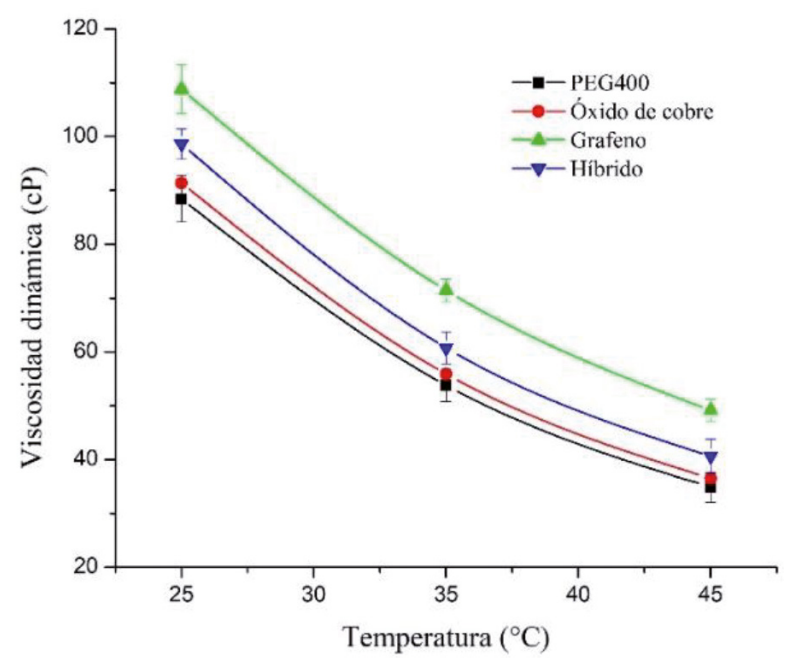

Figura 12. Viscosidad en función de la temperatura para las concentraciones de $0.1 \% \mathrm{p} / \mathrm{p}$.

Los valores obtenidos de viscosidad para el fluido base (PEG400) son similares a los obtenidos por Marcos y col., (2018). La viscosidad para los nanolubricantes con una concentración de $0.1 \% \mathrm{p} / \mathrm{p}$ no presentaron diferencias significativas con respecto a la viscosidad del PEG400.

Para la concentración de $0.5 \% \mathrm{p} / \mathrm{p}$ el nanolubricante PEG400-Grafeno y PEG400-CuO/Grafeno presentaron un incremento en la viscosidad a la temperatura de $45^{\circ} \mathrm{C}$ de $40.7 \%$ y $16.4 \%$ respectivamente. Las viscosidad del fluido base PEG400 aumentó con el incremento en la concentración de nanomaterial, estos resultados concuerdan con los reportados por Rostami et al., (2020)the rheological behavior of antifreeze containing a grouping of graphene oxide (GO. Este efecto surge debido a que los nanomateriales aumentan el orden molecular en el fluido aumentado así la viscosidad del mismo (Liñeira et al., 2018).

\section{Conclusiones}

- El nanolubricante PEG400-Grafeno presentó la mayor estabilidad en el tiempo y el comportamiento fue similar para las dos concentraciones utilizadas. Mientras que los nanolubricantes preparados con $\mathrm{CuO}$ presentaron una alta sedimentación.

- La conductividad térmica del nanolubricante PEG400-Grafeno $\quad(0.5 \% \mathrm{p} / \mathrm{p})$ fue $13.5 \%$ $\left(35^{\circ} \mathrm{C}\right)$ superior a la del PEG400. Este aumento es significativo teniendo en cuenta la baja concentración de nanoparticulas utilizadas.
- La viscosidad para los nanolubricantes a una concentración de $0.1 \% \mathrm{p} / \mathrm{p}$ no presentó diferencias significativas con respecto a la viscosidad del PEG400. Sin embargo, al incrementar la concentración hasta $0.5 \% \mathrm{p} / \mathrm{p}$ La viscosidad fue $40.7 \%$ superior para el nanolubricante PEGGrafeno con respecto a la del PEG400 a $45^{\circ} \mathrm{C}$.

\section{Agradecimientos}

Los autores desean agradecer al Instituto Tecnológico Metropolitano por apoyo financiero a través del programa de jóvenes investigadores e innovadores (Resolución No.360 de 06 ABR 2020, Resolución $\mathrm{N}^{\circ} 534$ de 19 JUN 2020 y Resolución N 863 de 08 OCT 2020).

\section{Referencias}

Akilu, S., Baheta, A. T., \& Sharma, K. V. (2017). Experimental measurements of thermal conductivity and viscosity of ethylene glycol-based hybrid nanofluid with $\mathrm{TiO} 2-\mathrm{CuO} / \mathrm{C}$ inclusions. Journal of Molecular Liquids, 246, 396-405. https://doi.org/10.1016/j.molliq.2017.09.017

Alemdar, A., Güngör, N., Ece, O. I., \& Atici, O. (2005). The rheological properties and characterization of bentonite dispersions in the presence of nonionic polymer PEG. Journal of Materials Science, 40(1), 171-177. https://doi.org/10.1007/s10853005-5703-4

Azman, N. F., \& Samion, S. (2019). Dispersion Stability and Lubrication Mechanism of Nanolubricants: A Review. International Journal of Precision Engineering and Manufacturing Green Technology, 6(2), 393-414. https://doi. org/10.1007/s40684-019-00080-x

Babar, H., \& Ali, H. M. (2019). Towards hybrid nanofluids: Preparation, thermophysical properties, applications, and challenges. In Journal of Molecular Liquids (Vol. 281, pp. 598-633). https://doi.org/10.1016/j.molliq.2019.02.102

Bhaumik, S., Kamaraj, M., \& Paleu, V. (2020). Tribological analyses of a new optimized gearbox biodegradable lubricant blended with reduced graphene oxide nanoparticles. Proceedings of the Institution of Mechanical Engineers, Part J: Journal of Engineering Tribology. https://doi. org $/ 10.1177 / 1350650120925590$

Chen, J., Spear, S. K., Huddleston, J. G., \& Rogers, R. D. (2005). Polyethylene glycol and solutions of polyethylene glycol as green reaction media. Green 
Chemistry, 7(2), 64-82. https://doi.org/10.1039/ b413546f

Cortes, V., Sanchez, K., Gonzalez, R., Alcoutlabi, M., \& Ortega, J. A. (2020). The performance of $\mathrm{SiO} 2$ and $\mathrm{TiO} 2$ nanoparticles as lubricant additives in sunflower oil. Lubricants, 8(1). https://doi. org/10.3390/lubricants8010010

Darminesh, S. P., Sidik, N. A. C., Najafi, G., Mamat, R., Ken, T. L., \& Asako, Y. (2017). Recent development on biodegradable nanolubricant: A review. International Communications in Heat and Mass Transfer, 86(June), 159-165. https://doi. org/10.1016/j.icheatmasstransfer.2017.05.022

Dhanola, A., \& Garg, H. C. (2020a). Influence of different surfactants on the stability and varying concentrations of $\mathrm{TiO} 2$ nanoparticles on the rheological properties of canola oilbased nanolubricants. Applied Nanoscience (Switzerland), $0123456789 . \quad$ https://doi. org/10.1007/s13204-020-01467-y

Dhanola, A., \& Garg, H. C. (2020b). Materials Today : Proceedings Experimental analysis on stability and rheological behaviour of TiO 2 / canola oil nanolubricants. Materials Today: Proceedings, $x x x x$. https://doi.org/10.1016/j.matpr.2020.04.245

Geng, Y., Boshra, A. A. A. A. A., Alsagri, A. S., Shahsavar, A., \& Talebizadehsardari, P. (2019). Characterization of the nanoparticles, the stability analysis and the evaluation of a new hybrid nanooil thermal conductivity. Journal of Thermal Analysis and Calorimetry, 0123456789. https:// doi.org/10.1007/s10973-019-08434-y

Gulzar, M., Masjuki, H., Varman, M., Kalam, M., Mufti, R. A., Zulkifli, N., Yunus, R., \& Zahid, R. (2015). Improving the AW/EP ability of chemically modified palm oil by adding $\mathrm{CuO}$ and $\mathrm{MoS} 2$ nanoparticles. Tribology International, 88, 271279. https://doi.org/10.1016/j.triboint.2015.03.035

Hemmat Esfe, M., Esfandeh, S., \& Rejvani, M. (2018). Modeling of thermal conductivity of MWCNT$\mathrm{SiO} 2(30: 70 \%) / E G$ hybrid nanofluid, sensitivity analyzing and cost performance for industrial applications: An experimental based study. Journal of Thermal Analysis and Calorimetry, 131(2), 1437-1447. https://doi.org/10.1007/s10973-017$\underline{6680-y}$

Hou, X., Jiang, H., Ali, M. K. A., Liu, H., Su, D., \& Tian, Z. (2020). Dispersion behavior assessment of the molybdenum disulfide nanomaterials dispersed into poly alpha olefin. Journal of Molecular Liquids, 311. https://doi.org/10.1016/j. molliq.2020.113303
Hu, Y., Wang, Y., Zeng, Z., Zhao, H., Ge, X., Wang, K., Wang, L., \& Xue, Q. (2018). PEGlated graphene as nanoadditive for enhancing the tribological properties of water-based lubricant. Carbon, 137, 41-48. https://doi.org/10.1016/j. carbon.2018.05.009

Huo, M., Wu, H., Xie, H., Zhao, J., Su, G., Jia, F., Li, Z., Lin, F., Li, S., Zhang, H., \& Jiang, Z. (2020). Understanding the role of waterbased nanolubricants in micro flexible rolling of aluminium. Tribology International, 151(April), 106378. https://doi.org/10.1016/j. triboint.2020.106378

Kim, H. J., \& Kim, D. E. (2015). Water lubrication of stainless steel using reduced graphene oxide coating. Scientific Reports, 5(C), 1-13. https://doi. org/10.1038/srep17034

Koshy, C. P., Rajendrakumar, P. K., \& Thottackkad, M. V. (2015). Evaluation of the tribological and thermo-physical properties of coconut oil added with MoS2 nanoparticles at elevated temperatures. Wear, 330-331, 288-308. https://doi.org/10.1016/j. wear.2014.12.044

Kotia, A., Rajkhowa, P., Rao, G. S., \& Ghosh, S. K. (2018). Thermophysical and tribological properties of nanolubricants: A review.

León, A., Reuquen, P., Garín, C., Segura, R., Vargas, P., Zapata, P., \& Orihuela, P. A. (2017). FTIR and raman characterization of $\mathrm{TiO} 2$ nanoparticles coated with polyethylene glycol as carrier for 2-methoxyestradiol. In Applied Sciences (Switzerland) (Vol. 7, Issue 1). https://doi. org/10.3390/app7010049

Liñeira, J. M., Guimarey, M. J. G., Comuñas, M. J. P., López, E. R., Amigo, A., \& Fernández, J. (2018). Thermophysical and tribological properties of dispersions based on graphene and a trimethylolpropane trioleate oil. Journal of Molecular Liquids, 268, 854-866. https://doi. org/10.1016/j.molliq.2018.07.107

Marcos, M. A., Cabaleiro, D., Guimarey, M. J. G., Comuñas, M. J. P., Fedele, L., Fernández, J., \& Lugo, L. (2018). PEG 400-based phase change materials nano-enhanced with functionalized graphene nanoplatelets. Nanomaterials, 8(1). https://doi.org/10.3390/nano8010016

Marcos, M. A., Podolsky, N. E., Cabaleiro, D., Lugo, L., Zakharov, A. O., Postnov, V. N., Charykov, N. A., Ageev, S. V., \& Semenov, K. N. (2019). MWCNT in PEG-400 nanofluids for thermal applications: A chemical, physical and thermal approach. Journal of Molecular Liquids, 294, 111616. https://doi. org/10.1016/j.molliq.2019.111616 
Mehrali, M., Sadeghinezhad, E., Latibari, S. T., Kazi, S. N., Mehrali, M., Zubir, M. N. B. M., \& Metselaar, H. S. C. (2014). Investigation of thermal conductivity and rheological properties of nanofluids containing graphene nanoplatelets. Nanoscale Research Letters, 9(1), 15. https://doi. org/10.1186/1556-276X-9-15

Mello, V. S., Faria, E. A., Alves, S. M., \& Scandian, C. (2020). Enhancing Cuo nanolubricant performance using dispersing agents. Tribology International, 150(March), 106338. https://doi.org/10.1016/j. triboint.2020.106338

Nabil, M. F., Azmi, W. H., Hamid, K. A., Zawawi, N. N. M., Priyandoko, G., \& Mamat, R. (2017). Thermophysical properties of hybrid nanofluids and hybrid nanolubricants: A comprehensive review on performance. International Communications in Heat and Mass Transfer, 83(March), 30-39. https:// doi.org/10.1016/j.icheatmasstransfer.2017.03.008

Osama, M., Walvekar, R., Khalid, M., Khaliq, A., Yin, W., Chandra, T., \& Manikyam, S. (2018). Physical properties optimization of POME-groundnutnaphthenic based graphene nanolubricant using response surface methodology. Journal of Cleaner Production, 193, 277-289. https://doi. org/10.1016/j.jclepro.2018.05.070

Pownraj, C., \& Valan Arasu, A. (2020a). Effect of dispersing single and hybrid nanoparticles on tribological, thermo-physical, and stability characteristics of lubricants: a review. In Journal of Thermal Analysis and Calorimetry (Issue 0123456789). Springer International Publishing. https://doi.org/10.1007/s10973-020-09837-y

Pownraj, C., \& Valan Arasu, A. (2020b). Effect of dispersing single and hybrid nanoparticles on tribological, thermo-physical, and stability characteristics of lubricants: a review. In Journal of Thermal Analysis and Calorimetry (Issue 0123456789). Springer International Publishing. https://doi.org/10.1007/s10973-020-09837-y

Rostami, S., Ahmadi Nadooshan, A., \& Raisi, A. (2020). The effect of hybrid nano-additive consists of graphene oxide and copper oxide on rheological behavior of a mixture of water and ethylene glycol. Journal of Thermal Analysis and Calorimetry, 139(3), 2353-2364. $\quad$ https://doi.org/10.1007/ s10973-019-08569-y

Sarkar, J., Ghosh, P., \& Adil, A. (2015). A review on hybrid nanofluids: Recent research, development and applications. Renewable and Sustainable Energy Reviews, 43, 164-177. https://doi. org/10.1016/j.rser.2014.11.023
Soni, S., \& Agarwal, M. (2014). Lubricants from renewable energy sources - a review. Green Chemistry Letters and Reviews, 7(4), 359-382. https://doi.org/10.1080/17518253.2014.959565

Suthar, K., Singh, Y., Surana, A. R., Rajubhai, V. H., \& Sharma, A. (2020). Experimental evaluation of the friction and wear of jojoba oil with aluminium oxide (A12O3) nanoparticles as an additive. Materials Today: Proceedings, 25, 699-703. https://doi.org/10.1016/j.matpr.2019.08.150

Wu, H., Jia, F., Zhao, J., Huang, S., Wang, L., Jiao, S., Huang, H., \& Jiang, Z. (2019). Effect of water-based nanolubricant containing nano-TiO 2 on friction and wear behaviour of chrome steel at ambient and elevated temperatures. Wear, 426-427(September 2018), 792-804. https://doi. org/10.1016/j.wear.2018.11.023

Yapici, K., Cakmak, N. K., Ilhan, N., \& Uludag, Y. (2014). Rheological characterization of polyethylene glycol based $\mathrm{TiO} 2$ nanofluids. Korea Australia Rheology Journal, 26(4), 355-363. https://doi.org/10.1007/s13367-014-0041-1

Yu, W., \& Xie, H. (2012). A review on nanofluids: Preparation, stability mechanisms, and applications. Journal of Nanomaterials, 2012. https://doi.org/10.1155/2012/435873

Zhang, C., Zhang, S., \& Song, S. (2014). Preparation and Tribological Properties of Surface-Capped Copper Nanoparticle as a Water-Based Lubricant Additive. 25-33. https://doi.org/10.1007/s11249014-0304-5

Fecha de recepción: 04 de diciembre de 2020 Fecha de aceptación: 26 de junio de 2021 